\title{
Gap Analysis between Provisional Diagnosis on Admission and Final Diagnosis during Discharge-A Comparative Study
}

\author{
Amitabha Chattopadhyay ${ }^{1}$, Ritu Ghosh ${ }^{2}$, Tanima Das ${ }^{3}$, \\ Sandipan Chakroborty ${ }^{4}$, Subhadeep Paul ${ }^{5}$, Saibendu Kumar Lahiri ${ }^{6}$ \\ 1,2(Assistant Professor, Department of Community Medicine, R.G.Kar Medical College, West Bengal, India) \\ 3(Associate Professor, Department of Medicine, IPGMER, West Bengal, India) \\ 4(M.D-PGT, Dept. of Medicine, R.G.KAR Medical College, Kolkata) \\ 5(Assistant Professor, Department of Community Medicine, Malda Medical College, West Bengal, India) \\ 6(Prof \& Head, Dept. of Community Medicine, R.G.KAR Medical College, Kolkata)
}

\begin{abstract}
Background: Achieving a high degree of diagnostic accuracy is important in practice of medicine. High degree of accuracy of provisional diagnosis may lead to use of lesser number of investigations with lesser cost burden to the health sector. Therefore, this gap analysis study aims to find out that how accurately provisional diagnosis matches final diagnosis. Methodology: This was a retrospective record based comparative study done in the Medicine Ward of R.G.Kar Medical College, Kolkata over a period of one month. Every patient having planned discharged on a particular day from malelfemale ward were selected and his/her treatment file was viewed and relevant informations were collected. The major outcome variable of the study was the matching/unmatching of final diagnosis with provisional diagnosis of the case. Results: Out of 420 patients, 182(43.3\%) had their final diagnosis same as that of their provisional diagnosis. Association was found to be significant in case of period of hospital stay, referrals, type of investigations and number of investigations. Clinical examination had $55.4 \%$ sensitivity and while diagnostic investigations had sensitivity of $44.51 \%$. Conclusion: Matching of provisional diagnosis with discharge diagnosis with greater accuracy and lesser number of investigations can lead to greater patient satisfaction along with lesser burden on health resources of the state. Technological developments for diagnosis are important but will never supplant the role of careful history taking for symptoms and clinical examination for signs.
\end{abstract}

Keywords - gap analysis, provisional diagnosis, final diagnosis

Submitted date 19 June 2013

Accepted Date: 24 June 2013

\section{Introduction}

A diagnosis is meant to provide a reliable description of the clinical condition, one that provides a means of communication between patient and the clinician, as well as interested patient parties. A provisional diagnosis is either the first considered diagnosis, or a subsequent diagnosis after the previous one has been found wanting. Nevertheless, the provisional diagnosis sets in motion the first stage of treatment and illness management. History taking, clinical examination and diagnostic investigations helps the clinician to come to the final diagnosis. Therefore to confirm the provisional diagnosis, one should proceed with available methods of investigations. The common methods are- Biochemical investigations, histopathological investigations, microbiological examinations, radiological investigations etc. The final diagnosis can usually be reached following chronological organization and critical evaluation of the information obtained from the patient history, physical examination and the result of radiological and laboratory examination. The final diagnosis usually identifies the diagnosis for the patient's primary complaint first, with subsidiary diagnosis of concurrent problems ${ }^{1}$.

Achieving a high degree of diagnostic accuracy is important in practice of medicine. Being able to make accurate diagnoses help to foster good doctor patient relationships and to build up rapport needed in subsequent management. High degree of diagnostic accuracy can have clinical, financial, legal implications. High degree of accuracy of provisional diagnosis may lead to use of lesser number of investigations with lesser cost burden to the health sector but providing greater satisfaction to the patients in terms of diagnosis and may lesser period of stay in hospital .Therefore, this gap analysis study aims to find out that how accurately provisional diagnosis matches final diagnosis and role of clinical examination and diagnostic investigations for such purpose. 


\section{Materials and Methods}

This was a retrospective record based comparative study done in the Medicine Ward of R.G.Kar Medical College, Kolkata over a period of one month (November 2010) which was selected by simple random sampling method. Every patient having planned discharged on a particular day from male/female ward were selected and his/her treatment file was viewed and relevant informations regarding the provisional diagnosis of the patient, investigations and final diagnosis during discharge were obtained and recorded in predesigned pretested schedule .The total number of discharge files reviewed were 439,out of which 19 case files did not have any provisional diagnosis mentioned in them. Before starting the study, ethical clearance for conducting the study was taken from the ethical committee of R.G.Kar Medical College, Kolkata. Informed consent of visiting physician and of the patient were also taken before recording of informations.

Predesigned and pretested schedules were used to record informations from the case files of the discharged patients. Informations were recorded regarding age, gender, period of stay in hospital, history of previous admission, details about provisional diagnosis and final diagnosis, number of referrals, types of investigations done and regarding the total number of investigations carried out. Opinion of treating physician was also recorded regarding whether clinical examination or diagnostic investigations were responsible for clinching the final diagnosis. The major outcome variable of the study was the matching/unmatching of final diagnosis with provisional diagnosis of the case. Matching was considered if final diagnosis was same as the provisional diagnosis or if it belonged to one of the differential diagnosis. Types of investigations were divided into four categories-biochemical test, pathology, imaging studies and cardiological tests/others. Statistical analysis was done by using Microsoft Excel 8.0 and Epi info 7 software. Chi-squared test was used for hypothesis testing; $p$ value $\leq 0.05$ was taken as level of significant association. Validity of clinical examination and diagnostic investigations as tool for matching of final diagnosis and provisional diagnosis was also determined by appropriate statistical software.

\section{Results}

Table 1 shows the baseline information as obtained from selected case files. Out of 439 case files reviewed, 19 were left out as no provisional or differential diagnosis was present in them. Out of 420 patients, 182(43.3\%) had their final diagnosis same as that of their provisional diagnosis (as depicted in figure 1), while in 238 cases the diagnosis did not match.

Table 2 represents the distribution of patients with matched/unmatched final and provisional diagnosis in relation to selected variables like age, gender, period of stay in hospital, history of previous admission, number of referrals, types of investigations and total number of investigations. It was found that in both $<60$ years and $\geq$ 60 years age group i.e the geriatric age group, the disagreement between final diagnosis and provisional diagnosis was more than $50 \%$. Similarly, in case of both gender, disagreement of diagnosis was more. Majority of patients with hospital stay of $>7$ days, had different final diagnosis $(75.9 \%)$, whereas $55.9 \%$ of the patient with stay of $<7$ days had agreement of diagnosis. Disagreement of diagnosis was present in more than $50 \%$ of the patients irrespective of whether history of previous admission was present or not. Matching of diagnosis was there in $30.2 \%$ of patients who had referrals, while it was $51.5 \%$ in patients who did not have referrals. Type of investigations $(\leq 2)$ was predominantly found in patients with matched diagnosis, while more than two type of investigation was mainly done in cases were diagnosis did not match. Patients who had more than 7 investigations done, predominantly belonged to the group of patient with disagreement of diagnosis $(72.1 \%)$. Association was found to be significant in case of period of hospital stay, referrals, type of investigations and number of investigations.

Table 3 shows the validity of clinical examination and diagnostic investigations as tool for matching final diagnosis and provisional diagnosis. Clinical examination had 55.4\% sensitivity and $66.03 \%$ specificity while diagnostic investigations had sensitivity of $44.51 \%$ and specificity of $36.39 \%$. The positive likelihood ratio for clinical examination was 1.5 and for diagnostic investigation was 0.71 .

Table 4 shows the distribution of different types of investigations used. In patients were final diagnosis matched provisional diagnosis, $28.6 \%$ of patients had all 4 type of investigations done, while $22.0 \%$ had biochemical test \& imaging as mode of investigation. In patients with different final diagnosis, 30.3\% of the patients had all 4 types of investigations done and $46.2 \%$ patients had biochemical test, pathology and imaging as mode of investigation.

\section{Discussion}

Gap analysis studies between admission diagnosis and discharge diagnosis are rare. Some studies regarding methods of diagnosis has been done in relation to specific diseases or related situations, but general comparison between final and initial diagnosis irrespective of specific disease situations in a hospital is a very rare attempt. Attempt to use clinical examination and proper history taking for accurate diagnosis has been highlighted in some studies but ideal situation of achieving maximum accuracy is still wanted. 
Few salient features of this study were that in $43.3 \%$ of the cases there was an agreement in diagnosis between the final diagnosis and provisional diagnosis. One such study in relation to matching of diagnosis was carried out in Hong Kong ,to find out the accuracy of emergency department admission and effect of investigations on diagnostic accuracy; of all admission diagnoses $71.4 \%$ matched the final diagnoses in this study $^{2}$. A study was also done in Lahore were $80 \%$ of the cases had correct provisional diagnosis ${ }^{3}$. No significant association was found between the matching /unmatching of final and provisional diagnosis with age and gender but period of hospitalization showed significant association, reason of which may have been that complicated cases where diagnosis did not match, may have required longer period of stay. Again, there was significant association in respect to referrals, as difficult cases may have required referrals by which the diagnosis may have been proved to be different from initial one. Similar reason may have been responsible for significant association in case of $\geq 2$ type of investigations. Clinical examination had $55.4 \%$ sensitivity and likelihood ratio of 1.5 for matching of final diagnosis with provisional diagnosis while diagnostic investigations had sensitivity of $44.51 \%$ and likelihood ratio of 0.71 .

An Australian study compared the sensitivity of clinical examination to radiographs for detection of dental caries in molars and found clinical exam a higher sensitive test $(96 \% \text { vs } 58 \%)^{4}$. An American study in relation to lumbar spinal stenosis, showed that history taking and physical examination had a strong likelihood ratio of $\geq 2$ for detection of the problem ${ }^{5}$.

Certain limitations of the study were obvious as certain factors like criticalness of the disease, individual accuracy/precision of the doctors in making provisional and final diagnosis, individual influence of diagnostic investigations were not studied separately and thus were not taken into consideration for analysis. However all these confounding factors can be reviewed in depth for further analysis in future studies.

In summary, there is definite need for improvement of diagnostic accuracy; good clinical assessment techniques, including history taking and clinical examination, remain the most important and rewarding diagnostic tools in diagnostic accuracy for physicians. Thus it is fundamental for physicians to master the skill of history taking, clinical examination in an efficient manner for maximum diagnostic accuracy with minimum diagnostic interventions so as to provide greater cost benefit to health sector.

\section{Conclusion}

Matching of provisional diagnosis with discharge diagnosis with greater accuracy and lesser number of investigations can lead to greater patient satisfaction along with lesser burden on health resources of the state. Historically, diagnosis relied more on symptoms. With development of systematic methods of clinical examination more reliance was placed on signs. However major breakthroughs in diagnosis were achieved when medical technology provided a wide array of accurate and reliable laboratory and radiological investigations. Further refinements in diagnosis are going to be based on technological developments but will never supplant the role of careful history taking for symptoms and clinical examination for signs.

\section{References}

[1] Ghom Anil. Text Book of Oral Medicine. Jayapee Publications;2005. p 73.

[2]. Chui HS, Chan KF, Chung CH, Ma K, Au KW.A comparison of emergency department admission diagnoses and discharge diagnoses: retrospective study; Hong Kong Journal of emergency medicine, Vol 10(2)April 2003:70-75

[3]. Chaudhry Muhammad Rashid. Accuracy of provisional diagnosis made in accident and emergency department; The Professional Medical Journal, Vol 5(1)1998:63-67.

[4]. Fracaro MS, Seow WK, McAllan LH, Purdie DM. The sensitivity and specificity of clinical assessment compared with bitewing radiography for detection of occlusal dentin caries. Pediatr Dent. 2001 May-Jun;23(3):204-10.

[5]. Katz Jeffery N, Daglas M, Stucki G, Katz N P, Bayley J, Fossel AH, Chang LC, Lipson SJ. Degenerative Lumbar spine stenosisDiagnostc value of the History and Physical exam. Arthiritis \&Rheumatism, Vol 35(9)September 1995:1236-41.

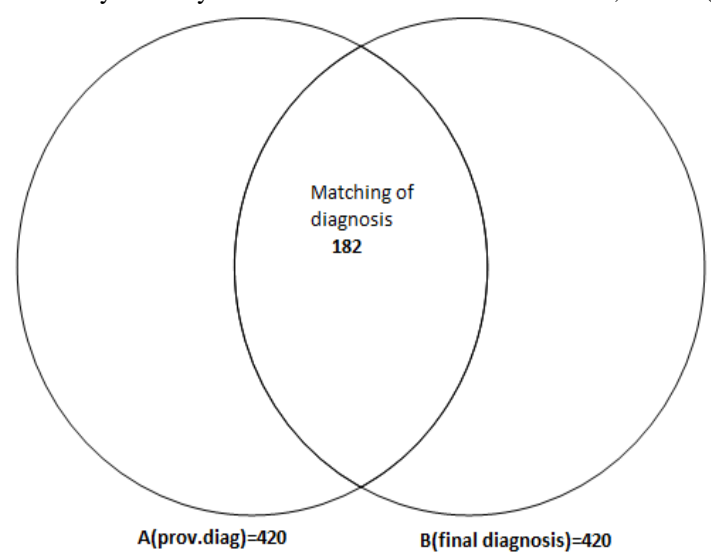

Figure 1: Venn diagram showing agreement between final diagnosis and provisional 
diagnosis in the overlapping zone.

Table-1 : Baseline information as obtained from selected case files.
Baseline information
Total no of case file reviewed
Provisional diagnosis/differential diagnosis present
Results
439
Number of case file not included (as no initial diagnosis present)
Final diagnosis matched with provisional/differential diagnosis $(n=420)$
Final diagnosis different from provisional/differential diagnosis $(n=420)$
$420(95.67 \%)$
19
$182(43.3 \%)$
$238(56.7 \%)$
7 days
Period of stay in hospital per patient(Median value) $(n=420)$
Number of investigations per patient(Median value) $(n=420)$
48.7 years

Table-2: Distribution of patients with matched/unmatched final and provisional diagnosis in relation to selected variables $(n=420)$

\begin{tabular}{|c|c|c|c|}
\hline \multirow{2}{*}{\multicolumn{4}{|c|}{ Final diagnosis donot match prov diag. $(\%)$ p value }} \\
\hline & & & \\
\hline$<60 \mathrm{yrs}$ & $124(42.9)$ & $165(58.1)$ & 0.88 \\
\hline$\geq 60 \mathrm{yrs}$ & $58(44.3)$ & $73(55.7)$ & \\
\hline \multicolumn{4}{|l|}{ 2)Gender } \\
\hline Male & $77(41.8)$ & $107(58.2)$ & 0.66 \\
\hline Female & $105(44.5)$ & $131(55.5)$ & \\
\hline \multicolumn{4}{|c|}{ 3)Period of stay } \\
\hline$\leq 7$ days & $142(55.9)$ & $112(44.1)$ & $\leq 0.05$ \\
\hline$>7$ days & $40(24.1)$ & $126(75.9)$ & \\
\hline \multicolumn{4}{|c|}{ 4)Previous admission } \\
\hline Present- & $42(42.8)$ & $56(57.2)$ & 0.99 \\
\hline Absent- & $140(43.5)$ & $182(56.5)$ & \\
\hline \multicolumn{4}{|l|}{ 5)Referrals } \\
\hline Yes- & $49(30.2)$ & $113(69 . .8)$ & $\leq 0.05$ \\
\hline No- & $133(51.5)$ & $125(48.5)$ & \\
\hline \multicolumn{4}{|c|}{ 6)type of investigations } \\
\hline$\leq 2$ & $59(80.8)$ & $14(19.1)$ & $\leq 0.05$ \\
\hline$>2$ & $123(35.4)$ & $224(64.6)$ & \\
\hline \multicolumn{4}{|c|}{ 7) number of investigations } \\
\hline$\leq 7$ & $134(54.0)$ & $114(46.0)$ & $\leq 0.05$ \\
\hline$>7$ & $48(27.9)$ & $124(72.1)$ & \\
\hline
\end{tabular}

Table-3: Validity of clinical examination and diagnostic investigations as tool for matching final diagnosis and provisional diagnosis. $(n=420)$

\begin{tabular}{|lccc|}
\hline $\begin{array}{l}\text { Tool for matching } \\
\text { final Diagnosis \& prov diag. }\end{array}$ & Sensitivity $(\%)$ & Specificity(\%) & Likelihood ratio \\
1)Clinical examination & 55.49 & 66.03 & 1.5 \\
& 44.51 & 36.39 & 0.71 \\
\hline
\end{tabular}

Table 4: Distribution of different types of diagnostic investigations used. $(\mathrm{n}=\mathbf{4 2 0})$

\begin{tabular}{|c|c|c|}
\hline $\begin{array}{l}\text { Tests } \\
\text { prov.diagnosis }(\%)\end{array}$ & $\begin{array}{l}\text { Final diagnosis matched } \\
\text { prov.diagnosis }(\%)\end{array}$ & Final diag. did not match \\
\hline Biochemistry- & $5(2.7)^{1}$ & $1(0.4)$ \\
\hline Biochem+pathology- & $12(6.6)$ & $1(0.4)$ \\
\hline Biochem+imaging- & $40(22.0)$ & $9(3.8)$ \\
\hline Biochem+cardio/others- & $2(1.1)$ & $3(1.3)$ \\
\hline Biochem+patho+imaging- & $39(21.4)$ & $110(46.2)$ \\
\hline Biochem+patho+imaging+cardio/ & /others- 52(28.6) & $72(30.3)$ \\
\hline Biochem+imaging+cardio/others & $22(12.1)$ & $27(11.3)$ \\
\hline Biochem+patho+cardio/others- & $10(5.5)$ & $15(6.3)$ \\
\hline
\end{tabular}

N.B- No cases had pathology, imaging or cardio/others as sole method of investigation. 